\title{
Le lien entre la Loi sur le développement durable et la Loi sur la qualité de l'environnement : sa nature et sa portée
}

\author{
Denis Bourque ${ }^{a}$
}

RÉSUMÉ. L'unicité du droit environnemental québécois a fait un bond en avant en 2017. En effet, par l'intermédiaire d'un amendement à la Loi sur la qualité de l'environnement, l'Assemblée nationale donne un coup d'envoi à l'effectivité des principes de développement durable dont plusieurs se retrouvent à l'article 6 de la Loi sur le développement durable. L'actualisation de ces principes permet de mettre à jour les processus décisionnels et les instruments juridiques utilisés par l'État québécois pour rendre ses décisions, notamment celles reliées au régime d'autorisation environnementale. L'initiative législative prise par le Québec se répercute également sur le partage des champs de compétence prévus dans la Loi constitutionnelle de 1867 et sur l'articulation des formes de gouvernance qui en découlent.

\begin{abstract}
The uniqueness of environmental law in Quebec has taken a leap forward in 2017. Indeed, through an amendment to the Environment Quality Act, the National Assembly kicks off the environment effectiveness of the principles of sustainable development, many of which are found in section 6 of the Sustainable Development Act. The nature of these principles allows to update the analytical processes and legal instruments used by the Quebec government to make its decisions, particularly those related to the environmental authorization regime. Quebec's legislative initiative is also reflected in the division of powers provided for in the Constitution Act, 1867, and the resulting articulation of the means of "governance".
\end{abstract}

\section{Introduction}

En mars 2017, l’Assemblée nationale insérait une « disposition préliminaire » dans la Loi sur la qualité de l'environnement ${ }^{1}$. Cette " disposition préliminaire» établit un lien entre deux lois « pivot» du droit environnemental québécois, à savoir la Loi sur le développement durable (LDD) ${ }^{2}$ et la Loi sur la qualité de l'environnement $(\mathrm{LQE})^{3}$ (LDD - LQE). Ce changement entraîne des conséquences importantes, notamment pour la réalisation des grands projets ayant des incidences économiques. Nous verrons d'abord le contenu de cette disposition préliminaire afin de bien la situer dans l'ordonnancement juridique québécois. Ensuite, nous verrons les principales caractéristiques des lois en cause afin de saisir la portée du lien qui les unit. Enfin, nous examineronsl'application du lien LDD - LQE dans trois si-

a Professeur, LL. D., avocat, Université du Québec à Chicoutimi. tuations particularisées : le lac Saint-Jean, les oléoducs et la Banque de l'infrastructure du Canada. Ces trois «terrains d'application» nous feront prendre conscience à la fois de l'importance de l'avancée législative réalisée, mais aussi de la complexité reliée à l'effectivité du droit en cause.

\section{L'adoption d'une « disposition préliminaire » dans la Loi sur la qualité de l'environnement}

Cette disposition préliminaire se lit comme suit :

\section{DISPOSITION PRÉLIMINAIRE}

Les dispositions de la présente loi visent la protection de l'environnement de même que la sauvegarde des espèces vivantes qui y habitent, dans la mesure prévue par la loi. Elles favorisent la 
réduction des émissions de gaz à effet de serre et permettent de considérer l'évolution des connaissances et des technologies, les enjeux liés aux changements climatiques et à la protection de la santé humaine, ainsi que les réalités des territoires et des collectivités qui les habitent.

Elles affirment le caractère collectif et d'intérêt public de l'environnement, lequel inclut de manière indissociable les dimensions écologiques, sociales et économiques.

Les objectifs fondamentaux de cette loi font que la protection, l'amélioration, la restauration, la mise en valeur et la gestion de l'environnement sont d'intérêt général.

Elles assurent le respect des principes de développement durable, tels que définis dans la Loi sur le développement durable (chapitre D8.1.1) ainsi que la prise en compte des impacts cumulatifs ${ }^{4}$.

D'entrée de jeu, en adoptant cette disposition préliminaire, l'Assemblée nationale souligne clairement qu'elle attribue une portée considérable aux objectifs de la LQE, qu'elle adhère à d'importantes valeurs environnementales et sociétales et qu'elle entend promouvoir l'effectivité des principes de développement durable en précisant que les dispositions de la LQE « assurent le respect des principes de développement durable tels que définis dans la Loi sur le développement durable $»^{5}$. L'Assemblée nationale a profité de la modernisation de la LQE pour mieux intégrer les 16 principes de la LDD dans le régime d'autorisation environnementale des projets et des activités susceptibles d'affecter l'environnement ${ }^{6}$.

Ce lien favorise la cohérence en matière de droit environnemental québécois. Ce secteur du droit est actuellement marqué par une forme d'éclatement puisque, sur le plan québécois, plusieurs lois touchent à ce secteur du droit, compte tenu des champs de compétence attribués aux États fédérés canadiens (provinces). À noter que cette forme d'éclatement existe également au niveau de l'État fédéral puisque les deux ordres de gouvernement peuvent adopter des lois touchant les questions environnementales. Comme le rappelle la professeure Halley, la Cour suprême du Canada a précisé que l'environnement n'est pas un domaine de compétence législative en vertu du partage des champs de compétence prévu dans la Loi constitutionnelle de
18677. La professeure Halley précise que l'environnement est plutôt un « sujet diffus qui touche plusieurs domaines fédéraux et provinciaux. Par conséquent, la Constitution doit être interprétée de façon à accorder aux deux paliers de gouvernement des moyens de protéger l'environnement tout en maintenant la structure fédérative de la Constitution. Une disposition relative à l'environnement est constitutionnellement valide si elle relève de par son caractère véritable de l'une des compétences législatives attribuées à son auteur $»^{8}$.

Par conséquent, autant au niveau fédéral que provincial (Québec), de multiples lois touchent l'environnement et celles-ci sont valides dans la mesure où elles se raccrochent à l'un ou l'autre des champs de compétence prévus dans la Loi constitutionnelle de 18679.

\section{Les caractéristiques de la Loi sur le développement durable (LDD) et de la Loi sur la qualité de l'environnement (LQE)}

\subsection{Les caractéristiques de la Loi sur le développement durable}

L'objectif de la loi est " d'instaurer un nouveau cadre de gestion au sein de l'Administration afin que l'exercice de ses pouvoirs et de ses responsabilités s'inscrive dans la recherche d'un développement durable $»^{10}$.

Afin de préciser l'objectif qu'elle poursuit, cette loi ajoute que les mesures qu'elle contient « concourent plus particulièrement à réaliser le virage nécessaire au sein de la société face aux modes de développement non viable, en intégrant davantage la recherche d'un développement durable, à tous les niveaux et dans toutes les sphères d'intervention, dans les politiques, les programmes et les actions de l'Administration $»^{11}$.

Cette loi prévoit aussi une définition du développement durable, lequel s'entend "d'un développement qui répond aux besoins du présent sans compromettre la capacité des générations futures à répondre aux leurs. Le développement durable s'appuie sur une vision à long terme qui prend en compte le caractère indissociable des dimensions environnementale, sociale et économique des activités de développement $»^{12}$. 
Quant à son application, cette loi touche « le gouvernement, le Conseil exécutif, le Conseil du trésor, les ministères, de même que les organismes du gouvernement et les entreprises du gouvernement visés par la Loi sur le vérificateur général $\gg^{13}$.

Par conséquent, pour le moment, les municipalités, les commissions scolaires, les hôpitaux et les universités ne sont pas assujettis à la Loi sur le développement durable. Cependant, le gouvernement pourrait éventuellement, par décret, décider de les assujettir ${ }^{14}$.

Relativement aux municipalités, il importe de bien saisir la portée des propos de la Cour d'appel du Québec dans l'arrêt Wallot ${ }^{15}$ où elle souligne que la Loi sur les compétences municipales attribue aux municipalités des compétences touchant plusieurs domaines, dont celui portant sur l'environnement. Cette loi accorde aux municipalités un large pouvoir réglementaire en cette matière. Selon la Cour d'appel, les lois québécoises relatives à l'environnement, y compris la Loi sur le développement durable, « doivent se refléter dans la mise en œuvre de la Loi sur les compétences municipales ». Donc, les dispositions de la Loi sur les compétences municipales doivent être appliquées «à la lumière du corpus législatif québécois en matière environnementale », lequel comprend la Loi sur le développement durable ${ }^{16}$. Par conséquent, dans la mise en œuvre, entre autres, de leur compétence spécifique en matière d'environnement, les municipalités doivent réfléchir sur les techniques à utiliser pour tenir compte, d'une façon ou d'une autre, des principes de la Loi sur le développement durable.

Afin d'atteindre son objectif, la loi prévoit l'élaboration et l'application d'une stratégie gouvernementale qui contient des principes de développement durable ${ }^{17}$ auxquels s'ajoutent 16 autres principes prévus dans la loi elle-même ${ }^{18}$.

En plus des principes de développement durable comme moyen d'atteindre son objectif, la Loi sur le développement durable prévoit l'utilisation de trois autres catégories de moyens.

Premièrement, la rédaction de « plans d'action » par les entités visées par la loi (comprenant les ministères et les sociétés d'État ${ }^{19}$.

Deuxièmement, la création du poste de commissaire au développement durable, lequel doit, au moins une fois par année, faire part « de ses constatations et de ses recommandations ayant trait à l'application de la Loi sur le développement durable » et « de ses commentaires concernant les principes, les procédures ou les autres moyens employés en matière de développement durable par l'Administration au sens de la Loi sur le développement durable, ainsi que par les autres organismes et établissements assujettis à cette loi » ${ }^{20}$.

Troisièmement, l'insertion dans la Charte des droits et libertés de la personne d'un nouveau droit qui se lit comme suit :

46.1 Toute personne a droit, dans la mesure et suivant les normes prévues par la loi, de vivre dans un environnement sain et respectueux de la biodiversité ${ }^{21}$.

Les professeurs Paule Halley et Denis Lemieux expriment les commentaires suivants quant à la valeur et l'effectivité de ce droit :

Au Québec, la Charte des droits et libertés de la personne occupe une place privilégiée dans la hiérarchie des normes. L'introduction du droit à l'environnement dans ce texte équivaut donc à une proclamation solennelle du caractère fondamental de la protection environnementale ${ }^{22}$.

L'intention du législateur en introduisant l'article 46.1 dans la Charte québécoise peut également être un critère permettant d'assurer la validité et l'effectivité de ce droit. Tout nous laisse supposer qu'elle visait à permettre à tout citoyen de se plaindre du non-respect du droit à l'environnement. Cette large portée accorde à cet article une valeur importante ${ }^{23}$.

Comme telle, la protection de l'environnement constitue une valeur fondamentale et un principe directeur de l'action gouvernementale ${ }^{24}$.

[...] il (l'article 46.1) pourra faire sanctionner judiciairement l'omission d'agir de l'Administration par un jugement déclaratoire $[\ldots]^{25}$.

[...] l'adoption d'une stratégie de développement durable par le gouvernement québécois est de nature à amplifier l'utilisation de l'article 46.1 comme facteur d'interprétation des lois et règlements et comme facteur décisionnel ${ }^{26}$. 
Rappelons qu'en 2008, le juge Dutil, qui s'exprimait au nom de la Cour d'appel du Québec, soulignait la portée quasi constitutionnelle du droit prévu à l'article 46.1 :

Le droit à un environnement sain a récemment été investi d'une valeur quasi constitutionnelle puisqu'il est désormais inscrit à l'article 46.1 de la Charte des droits et libertés de la personne. En cas de doute dans l'interprétation d'un acte administratif, il ne faudrait pas privilégier celle qui serait contraire à l'objectif de préservation de l'environnement, lequel doit être poursuivi à tous les niveaux de gouvernement ${ }^{27}$.

Cette valeur fondamentale que constitue la protection de l'environnement véhiculée par l'article 46.1 de la Charte québécoise prend une acuité particulière étant donné le fait que la Cour suprême du Canada dans l'arrêt Baker28 a exprimé le principe selon lequel « toute autorité administrative est présumée tenir compte des valeurs fondamentales lorsqu'elle adopte une décision $»^{29}$.

Sur le plan des responsabilités ministérielles, notons qu'en plus d'assurer la protection de l'environnement, le ministre du Développement durable, de l'Environnement et de la Lutte contre les changements climatiques est également " chargé de coordonner l'action gouvernementale en matière dedéveloppement durable et de promouvoir le respect, particulièrement dans leur volet environnemental, des principes de développement durable auprès de l'Administration et du public $»^{30}$.

\subsection{La Loi sur la qualité de l'environnement (LQE)}

La LQE est un élément important du droit environnemental québécois et la « disposition préliminaire » nouvellement insérée au début de la LQE en rappelle la portée et l'étendue.

Au moins 82 règlements ont été édictés en vertu de cette loi, lesquels touchent plusieurs domaines d'activité dont, entre autres, la protection de l'environnement ${ }^{31}$, les attestations d'assainissement ${ }^{32}$, la protection et la réhabilitation des territoires ${ }^{33}$ la protection et la gestion des ressources en eau ${ }^{34}$, l'assainissement de l'atmosphère ${ }^{35}$ et la gestion des matières résiduelles ${ }^{36}$.
D’une façon particulière, la LQE encadre le processus d'évaluation environnementale des grands projets industriels au terme duquel le gouvernement adopte un décret autorisant ou refusant le projet ${ }^{37}$. Les décrets délivrés en vertu de la LQE sont des instruments juridiques privilégiés qui vont contribuer à « assurer le respect des principes de développement durable, tels que définis dans la Loi sur le développement durable »38. Nous allons assister à une nouvelle configuration des décrets afin que ceux-ci traduisent juridiquement les principes de développement durable dans des situations particularisées.

\section{$3 \quad$ L'application de la « disposition préliminaire » à des situations particularisées}

\subsection{Le lac Saint-Jean}

La problématique reliée aux droits conférés à la compagnie Rio Tinto Alcan (RTA) est un terrain d'application intéressant visant à démontrer la nécessité de reconfigurer le contenu de cet instrument juridique qu'on appelle « décret » par lequel le gouvernement québécois rend sa décision. Ce décret, délivré en vertu de la LQE, doit davantage démontrer le lien entre les principes et les valeurs contenus dans la Loi sur le développement durable et les droits qui peuvent être conférés notamment par la LQE et qui ont trait à l'utilisation de la ressource qu'est le lac Saint-Jean.

Rappelons qu'en 1985, le Bureau d'audiences publiques sur l'environnement (BAPE) tenait une audience publique relativement au Programme de stabilisation des berges du lac Saint-Jean ${ }^{39}$. Dans son rapport, le BAPE met en évidence la nécessité de « faire la lumière sur les droits, devoirs et pouvoirs d'Alcan et des riverains $\gg{ }^{40}$. Il ajoute que cette clarification «constitue le fondement d'une meilleure compréhension de la légitimité des gestes posés par chacun $»^{41}$. Il précise également que, de diverses manières, la « confusion » caractérise les droits conférés ainsi que l'exercice de ceux-ci ${ }^{42}$. Après avoir examiné sous plusieurs aspects la portée et l'étendue des droits conférés à RTA (autrefois Alcan) par l'État québécois, le BAPE pose les deux questions vitales suivantes : «Cette logique juridique a-t-elle encore un sens au Québec en 1985? Ne mériteraitelle pas d'être révisée? "43 $^{43}$ BAPE insiste sur le fait que différents problèmes juridiques devraient 
« être clarifiés, mis à jour et interprétés à la lumière des réalités sociales et juridiques de $1985 »^{44}$.

En vue de la tenue d'une audience publique par le BAPE à l'automne 2016 sur le Programme de stabilisation des berges du lac Saint-Jean 2017-2026, RTA a déposé une étude d'impact sur l'environnement ${ }^{45}$. Dans son étude d'impact, la compagnie Rio Tinto Alcan fait une description des droits qu'elle détient ${ }^{46}$. Dans le cadre législatif de l'époque, notamment en vertu d'un acte notarié du 12 décembre 1922 (publié le 28 juillet 1924) et de la Loi de la Commission du lac Saint-Jean (avril 1927), le gouvernement du Québec a octroyé à RTA plusieurs « catégories de droits », plus particulièrement celui « d'élever et de maintenir les eaux jusqu'au niveau maximum de 17,5 pieds au-dessus de zéro de l'échelle d'étiage du quai de Roberval » ${ }^{47}$. De plus, de gré à gré, « divers droits » ont été acquis depuis plusieurs décennies par RTA avec un grand nombre de riverains du lac Saint-Jean. Il s'agit de « divers droits autres que le droit de propriété ou nue-propriété que possède RTA » et qui ont trait notamment à l'inscription de « servitudes conventionnelles » dans des transactions privées avec des riverains.

Quant à la question des droits qu'elle détient, RTA résume la situation de la façon suivante:

Bref, les droits exercés par Rio Tinto Alcan aujourd'hui découlent principalement de l'acte de 1922, des droits acquis de gré à gré et de ceux issus du processus mis en place par la Loi de la Commission du lac Saint-Jean de 1927. L'exploitation des ressources hydrauliques se fait également conformément, entre autres, à la Loi sur le régime des eaux, en vigueur au Québec depuis fort longtemps ${ }^{48}$.

En terminant la description des droits qu'elle détient, RTA formule l'affirmation suivante :

Les droits historiquement concédés aux auteurs de Rio Tinto Alcan et exercés depuis près d'un siècle sont clairs. Ils font toutefois appel à des concepts juridiques nombreux et parfois fort complexes. Nous sommes convaincus que l'exercice de ces droits s'inscrit parfaitement dans le contexte juridique actuel ${ }^{49}$.

L'audience publique qui devait avoir lieu à l'automne 2016 a été reportée à mai 2017 afin de

Revue Organisations \& Territoires $\bullet$ Volume $26 \bullet \mathrm{N}^{\circ} 1-2 \bullet 2017$ permettre aux parties prenantes d'engager des négociations sur la gestion du lac Saint-Jean. Ces négociations ont permis aux parties prenantes d'adopter l'Entente de principe d'avril 2017 (Modèle de gestion participative du lac Saint-Jean) $)^{50}$.

À l'occasion de l'audience tenue en mai et juin 2017, le promoteur a de nouveau fait la description des droits qu'il détient ${ }^{51}$ et les autres parties prenantes ont fait part de leurs préoccupations ${ }^{52}$. Les points de vue exprimés portaient « tout d'abord sur les droits de RTA et des propriétaires riverains du lac Saint-Jean, la gestion participative du lac convenue dans l'Entente de principe d'avril 2017 et la fluctuation de son niveau d'eau au cours de l'année $»^{53}$.

Dans son rapport, le BAPE a pris acte, entre autres choses, de l'Entente de principe d'avril 2017 en formulant certains avis ${ }^{54}$. Quant à la problématique de l'ensemble des droits détenus par RTA (ceux conférés par l'État québécois et ceux acquis de gré à gré), le BAPE a dit qu'il appartenait à l'État québécois de décider s'il était opportun d'enclencher cette révision ${ }^{55}$.

Compte tenu des faits, des constats et des avis contenus dans les deux rapports du BAPE (celui de 1985 et celui de 2017), et prenant en considération la portée et l'étendue de la nouvelle disposition préliminaire de la LQE, l'État québécois devrait réviser l'attribution des droits conférés à RTA. Le Comité des parties prenantes de la gestion durable du lac Saint-Jean affirme qu'une « large majorité sinon la totalité des utilisateurs et riverains du lac Saint-Jean considèrent les droits de RTA excessifs en 2017, soit à toutes fins utiles celui d'éroder ses berges sans obligation aucune de corriger la situation. Le Comité demande au législateur de rendre le promoteur imputable des conséquences de sa gestion. Il ajoute que de nombreux propriétaires riverains remettent en cause les droits de propriété du promoteur et réclament de clarifier les droits et responsabilités de chacune des parties prenantes de la gestion du lac, de même que les droits et intérêts de la Première Nation des Pekuakamiulnuatsh $»^{56}$.

En fait, les motifs militant en faveur d'une telle révision trouvent des points d'appui principalement dans trois éléments. Premièrement, l'attribution actuelle des droits ne respecte pas le nouveau cadre juridique qui a été mis en place par le lien LDD - LQE, lequel est caractérisé par l'émergence de nouveaux concepts juridiques et de nouvelles 
valeurs sociétales (nouveaux paradigmes) qui conduisent, entre autres choses, à une nouvelle configuration de l'instrument juridique utilisé pour rendre la décision, à savoir le décret ${ }^{57}$.

Déjà en 2011 dans l'arrêt $W$ allot, la Cour d'appel du Québec soulignait l'influence de la LDD dans le processus décisionnel et l'émergence de nouvelles valeurs environnementales et de nouveaux concepts juridiques. Elle marque en ces termes cette évolution du cadre juridique :

Cette loi (Loi sur le développement durable) oblige l'administration publique, lors de l'exercice de ses attributions, tout en répondant aux besoins présents, de ne pas compromettre ceux des générations futures. Plus précisément, la loi prévoit que la protection de l'environnement fait partie du processus de développement durable et que l'ensemble des activités humaines doit être respectueux de la capacité de support des écosystèmes, d'où la nécessité d'en assurer la pérennité58.

D'autres concepts juridiques et valeurs environnementales et sociétales s'ajoutent à ceux déjà énoncés par la Cour d'appel, à savoir le « rôle de fiduciaire de l'État québécois notamment en matière d'utilisation des ressources naturelles ${ }^{59}$, le concept de " patrimoine commun $»^{60}$, le concept « d'État gardien $»^{61}$, le concept d'acceptabilité sociale $»^{62}$, le principe du «pollueur payeur $»^{63}$, le concept de «bien collectif $»^{64}$.

Dans l'arrêt Wallot, la Cour d'appel souligne l'importance de la Loi affirmant le caractère collectif des ressources en eau et visant à renforcer leur protection qui prévoit que l'eau est une ressource qui fait « partie du patrimoine commun de la nation québécoise »; elle attire notre attention sur l'article 2 de cette législation qui prévoit que « la protection, la restauration, la mise en valeur et la gestion des ressources en eau sont d'intérêt général et concourent à l'objectif de développement durable ${ }^{65}$.

Ces propos de la Cour d'appel prennent une acuité particulière dans le contexte où RTA souligne que " légalement, elle n'est nullement tenue de lutter contre l'érosion que ses activités pourraient causer $»^{66}$.

La détention excessive de droits par RTA, comme cela a été mentionné par le Comité des parties prenantes de la gestion durable du lac Saint-Jean, rend difficile la détermination des contours du concept de « patrimoine commun » (bien collectif) dans son application à la gestion du lac Saint-Jean. Pourtant, dans son étude d'impact, RTA semblait manifester une ouverture relativement au concept de "patrimoine commun ». En effet, relativement à la Loi affirmant le caractère collectif des ressources en eau et visant à renforcer leur protection, RTA affirme elle-même qu'il s'agit là «d'une loi importante dont la portée de certains nouveaux concepts qu'elle introduit reste à déterminer $»^{67}$. Elle souligne que «si éventuellement, par l'adoption de cette loi, Rio Tinto Alcan se voyait attribuer des responsabilités additionnelles envers le patrimoine commun, puisque l'État en est le gardien, il devrait à ce titre permettre à Rio Tinto Alcan de remplir ses obligations, en l'occurrence, dans le cadre d'un renouvellement de décret ${ }^{68}$. RTA admet donc la possibilité que l'État québécois puisse intervenir et modifier, d'une certaine manière, par décret ou par loi, les droits qu'elle détient compte tenu de l'évolution du contexte juridique québécois provoqué, entre autres, par la Loi affirmant le caractère collectif des ressources en eau et visant à renforcer leur protection. Compte tenu de ce contexte, il devient difficile de saisir l'affirmation de RTA selon laquelle «l'exercice de ces droits s'inscrit parfaitement dans le contexte juridique actuel » lorsque cette même compagnie admet, du même souffle, que le contexte juridique québécois contient de «nouveaux concepts $[\ldots]$ qu'il reste à déterminer $»^{69}$.

Deuxièmement, l'attribution actuelle des droits ne respecte pas les principes de développement durable. Dans son Programme de stabilisation des berges $d u$ lac Saint-Jean (2017-2026) - Étude d'impact sur l'environnement, RTA affirme ce qui suit : « Rio Tinto Alcan respecte en tous points les principes de développement durable auxquels réfère entre autres la Loi sur le développement durable. Le rapport d'analyse du développement durable du PSBLSJ de la Chaire en éco-conseil de l'Université du Québec à Chicoutimi (UQAC) le confirme $»^{70}$. À la demande de RTA, la Chaire en éco-conseil de l'UQAC a effectivement rédigé et remis un rapport à $\mathrm{RTA}^{71}$. Cependant, ce rapport ne confirme pas que RTA « respecte en tous points les principes de développement durable ». Sur la question précise de la clarification des droits détenus par RTA, la Chaire en éco-conseil affirme plutôt qu'il y a un " enchevêtrement des droits » et qu'en vertu des principes de développement durable, l'État québécois doit procéder à la clarification des droits détenus par $\mathrm{RTA}^{72}$. 
Troisièmement, l'attribution actuelle des droits ne respecte pas un droit de portée quasi constitutionnelle et un autre de portée constitutionnelle. Le droit de portée quasi constitutionnelle est prévu à l'article 46.1 de la Charte québécoise des droits et libertés de la personne ${ }^{73}$.

Dans le contexte de l'utilisation et de l'exploitation du lac Saint-Jean, la personne qui désire se prévaloir du droit que lui confère l'article 46.1 doit « s'aménager » et « cohabiter » avec ceux conférés à RTA. Cependant, pour cohabiter, encore faudrait-il connaittre la nature, l'étendue et la portée des droits conférés à RTA. La nature, la diversité et les catégories de droits décrits par RTA ne permettent pas à une personne de respecter la «mesure » et les «normes prévues par la loi ». Voici un exemple qui donne une idée de la complexité et du caractère bigarré de certains droits détenus par RTA : «Une participante indique que le droit de baignage du promoteur et la superficie de la servitude qui y est associée augmentent avec l'érosion et le recul des berges. Elle indique ainsi qu'il s'agit d'un "droit non seulement perpétuel et réel, mais qui se sert luimême" " ${ }^{74}$. Cet exemple démontre qu'il est difficile de saisir la portée de l'affirmation de RTA selon laquelle les droits qu'elle détient sont « clairs » mais « fort complexes $»^{75}$.

Cette confusion qui semble exister dans certaines catégories de droits détenus par RTA prend une acuité particulière compte tenu du fait que le BAPE exprime l'avis que « le promoteur et ses partenaires du futur Conseil de gestion durable du lac Saint-Jean gagneraient à produire et à diffuser un document conjoint de vulgarisation résumant les droits et obligations de Rio Tinto Alcan et des propriétaires riverains dans le contexte du programme de stabilisation des berges et de l'entente de principe d'avril

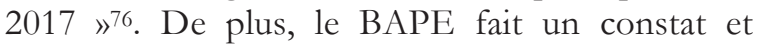
exprime l'avis suivant:

La commission d'enquête constate que l'entente d'avril 2017 ne précise pas comment la structure de gouvernance qu'elle prévoit s'intégrera au cadre préexistant de gestion intégrée des ressources en eau du bassin versant du lac Saint-Jean. [...] Avis - La commission d'enquête est d'avis qu'il faudrait prévoir l'arrimage des travaux de la structure de gouvernance avec ceux de la table de concertation de l'Organisme de bassin versant Lac-Saint-
Jean, notamment afin d'assurer la conformité de la nouvelle gouvernance du lac avec les orientations définies par cet organisme ${ }^{77}$.

Les remarques formulées par le BAPE auront des conséquences sur les conditions d'exercice du droit conféré à toute personne en vertu de l'article 46.1 de la Charte québécoise des droits et libertés de la personne. En d'autres termes, pour qu'une personne exerce son droit prévu à l'article 46.1, il devra se situer par rapport aux droits détenus par RTA qui, elle-même, devra établir la ligne de démarcation avec les droits détenus par les riverains. Cet exercice va inéluctablement se répercuter sur le fonctionnement de la structure de gouvernance prévue dans l'Entente de principe d'avril 2017. Et celle-ci devra, de son côté, trouver le moyen, sur le plan du droit, de " s'arrimer » avec la structure de gouvernance de l'organisme de bassin versant (OBV).

Encore ici, la portée excessive des droits conférés à RTA risque de rendre difficile de distinguer les droits qu'elle détient par rapport à ceux des riverains. Partant, cette difficulté pourrait compromettre le fonctionnement de la structure de gouvernance prévue à l'Entente de principe d'avril 2017 et empêcher d'intégrer celle-ci dans la structure de gouvernance de l'organisme de bassin versant.

Quant au droit de portée constitutionnelle, il est prévu à l'article 35 de la Loi constitutionnelle de 1982 et se lit comme suit: « Les droits existants - ancestraux ou issus de traités - des peuples autochtones du Canada sont reconnus et confirmés ». S'appuyant sur cet article, la Première Nation des Pekuakamiulnuatsh fait valoir ses droits ancestraux de la façon suivante :

Les droits consentis à la Quebec Development Company Limited, aujourd'hui Rio Tinto [Alcan], l'ont été sans égard aux droits ancestraux, y compris le titre aborigène, de notre Première Nation qui occupait déjà ce territoire depuis plus de 6000 ans. Il en a été de même lors de l'émission du premier décret en 1986 et son renouvellement en [1995] et en $2006^{78}$.

Donc, sur le plan constitutionnel, que ce soit au titre de l'article 46.1 de la Charte québécoise des droits et libertés de la personne ou au titre de l'article 35 de la Loi constitutionnelle de 1982, l'État québécois doit clarifier les titres conférés à RTA79. 
Le cadre juridique entourant la délivrance du décret conférant des droits à RTA a donc évolué, surtout avec le lien LDD - LQE. D'autres régimes juridiques ont récemment changé. Du côté des municipalités, par exemple, la Cour d'appel du Québec dans l'arrêt $W$ allot a modifié le cadre juridique de l'application de la Loi sur les compétences municipales en exigeant des municipalités, lorsqu'elles exercent leurs pouvoirs en vertu de cette loi, la prise en compte du « corpus législatif québécois en matière environnementale ». Pour sa part, la Cour suprême du Canada dans l'arrêt Ciment du Saint-Laurent a fait évoluer le cadre juridique de l'application de l'article 976 du CCQ en reconnaissant, en vertu de cet article, l'existence d'un régime de responsabilité civile sans faute en matière de troubles de voisinage qui serait fondé sur le caractère excessif des inconvénients subis par la victime et non sur le comportement de leur auteur présumé tout en précisant que ce nouveau régime de responsabilité contribue à la protection de l'environnement et à l'application du principe de pollueur payeur ${ }^{80}$.

Quant à l'Entente de principe d'avril 2017, le Comité des parties prenantes de la gestion durable du lac Saint-Jean qualifie cet accord "d'avancée majeure dans la reconnaissance du lac Saint-Jean comme une ressource collective qui doit être gérée par toute la collectivité, ce qui inclut Rio Tinto Alcan ${ }^{81}$. D'importantes modalités de fonctionnement restent à déterminer. Une étape importante a été franchie par les parties prenantes.

Cependant, d'autres gestes devront être posés afin d'effectuer le véritable virage demandé par la Loi sur le développement durable dont l'objectif est d'en arriver à une " gestion intégrée et concertée » du lac SaintJean, laquelle doit être " être effectuée en tenant compte des principes de développement durable, notamment ceux énoncés à l'article 6 de la $\mathrm{LDD} » 82$.

En ce qui a trait à l'octroi des droits hydrauliques qui affectent le grand bassin hydrographique du Saguenay-Lac-Saint-Jean, la professeure Marie-Claude Prémont s'exprime ainsi :

L'acquisition de droits hydrauliques d'une telle magnitude sur un grand bassin hydrographique ne s'est pas faite sans créer des conflits d'usage hier comme aujourd'hui, autant avec les autres projets industriels de la région qu'avec des droits individuels. [...] La tragédie du lac Saint-Jean illustre avec force le conflit d'usage entre l'occupation des berges du lac Saint-Jean et l'exploitation des droits hydrauliques. [...] Les débats et la recherche doivent se poursuivre quant à la conciliation des droits et l'impact social et économique de l'exploitation des droits hydrauliques au SLSJ ${ }^{83}$.

\subsection{Les oléoducs}

Notre deuxième situation particularisée (terrain d'application) soulève des questions reliées à l'applicabilité du lien LDD - LQE. Elle est reliée à la problématique de la construction des oléoducs au Canada. Rappelons qu'en novembre 2016, le gouvernement fédéral autorisait la construction de deux oléoducs ${ }^{84}$.

Étant donné que ces projets énergétiques traversent plusieurs provinces canadiennes (États fédérés canadiens), ils sont qualifiés de projets énergétiques fédéraux et, partant, sont assujettis aux dispositions de la Loi sur l'Office national de l'énergie85. En conséquence, c'est le processus d'évaluation environnementale fédéral qui s'applique et les décisions relatives à la réalisation des projets relèvent de l'État fédéral. Ainsi, les processus d'évaluation environnementale des États fédérés, y compris celui du Québec avec le lien LDD - LQE qui le caractérise, ne s'appliquent pas en dépit du fait que ces projets ont des répercussions importantes sur leur territoire respectif.

Le 13 janvier 2016, la Cour suprême de la ColombieBritannique rendait une décision dans l'affaire Coastal First Nations v. British Columbia (Environment) ${ }^{86}$ qui présente une avenue intéressante comme piste de solution. Cette affaire met en cause le projet de la compagnie Northern Gateway Pipelines Ltd (NGP) qui vise à construire un oléoduc entre Bruderheim, en Alberta et Kitimat, en Colombie-Britannique (d'une longueur de $660 \mathrm{~km}$ et traversant quelque 850 cours d'eau en Colombie-Britannique) ${ }^{87}$.

La décision de la Cour suprême de la ColombieBritannique met en cause la doctrine de l'exclusivité des compétences et la doctrine de la prépondérance fédérale. S'appuyant, entre autres choses, sur l'application de la doctrine de l'exclusivité des compétences et sur les principes du "fédéralisme coopératif », la Cour suprême de la Colombie-Britannique affirme que le projet de pipeline Northern Gateway Pipelines Ltd (NGP), bien qu'étant un 
projet fédéral, a des incidences sur les champs de compétence provinciaux et, qu'à ce titre, la Colombie-Britannique peut délivrer une autorisation environnementale (un décret) dans lequel elle peut inscrire des exigences plus sévères que celles formulées par le gouvernement fédéral. Le problème est de savoir jusqu'où la Colombie-Britannique peut aller dans ses exigences. La Cour suprême de la Colombie-Britannique exprime quand même l'avis qu'il y a un espace législatif et réglementaire qui peut être occupé par la province de la ColombieBritannique. L'ampleur de l'espace législatif et réglementaire doit être déterminée au cas par $\operatorname{cas}^{88}$.

Par conséquent, en s'appuyant, entre autres, sur les principes du "fédéralisme coopératif», les États fédérés canadiens ont le pouvoir d'appliquer leur processus d'évaluation environnementale et de délivrer, le cas échéant, des décrets qui pourraient contenir des exigences plus sévères que celles de l'État fédéral mais qui, par exemple, seraient de nature à protéger leurs terres agricoles et leurs cours d'eau.

Cependant, un autre contexte juridique pourrait se présenter et faire échec à l'ouverture faite aux États fédérés par l'arrêt Coastal. Les promoteurs des deux projets concernés, devant l'ampleur des obstacles juridiques à surmonter (comprenant l'ouverture faite aux provinces par l'arrêt Coastal), font des pressions sur le gouvernement fédéral ${ }^{89}$ pour que celui-ci exerce des pouvoirs très particuliers qu'il détient en vertu de la Loi constitutionnelle de 1867 afin de rendre inapplicables les lois provinciales vis-àvis de leurs projets. En effet, le Parlement fédéral pourrait exercer son pouvoir relatif à " l'intérêt national $»^{90}$, à « l'urgence » ${ }^{91}$ ou exercer son « pouvoir déclaratoire $»^{92}$.

Donc, pour offrir une solution au passage des pipelines, le gouvernement fédéral pourrait exercer les pouvoirs constitutionnels spéciaux qu'il détient. Cependant, il se produirait un problème de crédibilité qui, sur le plan politique, rendrait difficile l'exercice de tels pouvoirs constitutionnels prépondérants ${ }^{93}$.

D’une part, les autorités fédérales font preuve d'une certaine incohérence en approuvant, d'un côté, les deux pipelines en novembre 2016 et, d'un autre côté, en adoptant en décembre 2016 le Cadre pancanadien sur la croissance propre et les changements climatiques pour lutter contre les changements climatiques ${ }^{94}$.
D'autre part, en 2017, trois importants rapports d'experts ont été rendus publics dont le premier propose de revoir en profondeur le processus d'évaluation environnementale fédéral ${ }^{195}$, le deuxième recommande la modernisation radicale de l'Office national de l'énergie, tandis que le troisième met en évidence le fait que le gouvernement fédéral est loin d'être prêt à faire face à des tempêtes majeures, ainsi qu'à l'augmentation prévue du nombre d'inondations et d'incendies que risquent d'entrainer les changements climatiques ${ }^{96}$.

Le fonctionnement du gouvernement fédéral soulève donc le problème de sa crédibilité et de sa fiabilité. S'agissant d'une situation juridico-politique, le Québec (comme les autres États fédérés) peuvent exiger du gouvernement fédéral qu'il fasse preuve de retenue et laisse aux provinces la possibilité d'utiliser l'ouverture créée par l'arrêt Coastal. La solution juridique dégagée par l'arrêt Coastal doit être conservée, au premier chef, parce que ces projets énergétiques ont des répercussions colossales sur le territoire des États fédérés, menaçant, sous plusieurs angles, leur environnement et la qualité de vie de leurs citoyens ${ }^{97}$.

\subsection{La Banque de l'infrastructure du Canada (BIC)}

La troisième situation particularisée (terrain d'application) vise l'applicabilité du lien LDD - LQE aux projets réalisés par la Banque de l'infrastructure du Canada.

Dans le cadre de son plan Investir dans le Canada, le gouvernement fédéral effectue de nouveaux investissements dans les infrastructures " pour bâtir les villes du $21^{\text {e }}$ siècle et offrir aux collectivités de l'ensemble du pays les outils dont elles ont besoin pour prospérer et innover. À cette fin, le gouvernement fédéral investit plus de 180 milliards de dollars sur 12 ans pour 5 grandes priorités en matière d'infrastructures : les infrastructures du transport en commun, les infrastructures vertes, les infrastructures sociales, les infrastructures du commerce et du transport, et les infrastructures des collectivités rurales et nordiques ${ }^{98}$. L'un des mécanismes utilisés pour réaliser ces investissements prend la forme d'« ententes bilatérales intégrées » conclues avec chacun des États fédérés du Canada (provinces) ${ }^{99}$. 
L'autre moyen utilisé par le gouvernement fédéral est la création de la Banque de l'infrastructure du Canada. Le 22 juin 2017, le Parlement fédéral adoptait la Loi sur la Banque de l'infrastructure du Canada100 créant ainsi la Banque de l'infrastructure du Canada ${ }^{101}$ dont la mission est $4[\ldots]$ de faire des investissements et de chercher à attirer des investissements d'investisseurs du secteur privé et d'investisseurs institutionnels dans des projets d'infrastructures situés au Canada ou en partie au Canada qui généreront des recettes et qui seront dansl'intérêt public, par exemple en soutenant des conditions favorables à la croissance économique ou en contribuant à la viabilité de l'infrastructure au Canada »102. Parmi les fonctions que la banque peut remplir pour réaliser sa mission, notons qu'elle peut « exercer toute autre fonction utile à la réalisation de sa mission que le gouverneur en conseil peut préciser par décret $»^{103}$.

Parmi les pouvoirs conférés à la Banque de l'infrastructure du Canada, retenons qu'elle peut «conclure des ententes ou accords avec des ministères ou organismes fédéraux ou provinciaux, tout autre organisme ou toute autre personne et agir comme mandataire de ceux-ci, pour la prestation de services ou de programmes qui leur sont destinés ou qui sont fournis par eux, en leur nom ou conjointement avec eux, et fournir une aide financière en leur nom aux termes de l'entente ou de l'accord »104.

Notons que la banque a le statut de mandataire de Sa Majesté du Chef du Canada lorsqu'elle agit à titre de mandataire au sens de l'article $18 \mathrm{~h})^{105}$ ou lorsqu'elle «mène toute activité utile à la réalisation de sa mission que le gouverneur en conseil peut préciser par décret ${ }^{106}$.

Il convient de se demander si ce statut de mandataire de Sa Majesté du Chef du Canada permet à la Banque de l'infrastructure du Canada d'exclure l'application des lois provinciales dans l'exécution des fonctions que sa loi constitutive lui attribue. Selon le professeur Patrick Taillon, constitutionnaliste, cette disposition législative qui attribue le statut de mandataire de $\mathrm{Sa}$ Majesté du Chef du Canada à la Banque de l'infrastructure du Canada permet à certains partenariats "d'être soustraits à l'application d'une portion considérable des lois et règlements, même si les provinces et les municipalités étaient impliquées dans le choix des projets ». Le professeur Taillon ajoute que « mal- gré les intentions fédérales, la loi donne au gouvernement le pouvoir de décréter, au cas par cas, que la Banque agit comme mandataire de la Couronne. Il est possible qu'en pratique, le gouvernement fasse preuve de retenue dans l'application de la loi, mais juridiquement rien ne le force ou ne le limite à cette retenue $»^{107}$. Dans le même sens, l'Assemblée nationale a adopté une motion de façon unanime le 31 mai 2017 exigeant des amendements au projet de loi C- 44 « afin de démontrer que la Banque de l'infrastructure du Canada est soumise aux lois du Québec $»^{108}$.

En fait, en matière de conception, de gestion et de construction d'infrastructures, la BIC présente trois composantes majeures. Premièrement, le gouvernement fédéral n'hésite pas à affirmer qu'il « sera responsable d'établir l'orientation stratégique globale et les priorités en matière d'investissement de haut niveau pour la BIC $»^{109}$. Dans son budget 2017, le gouvernement fédéral ajoute que la nouvelle BIC « collaborera avec des partenaires d'investissement provinciaux, territoriaux, municipaux, autochtones et du secteur privé dans le but de transformer la façon dont l'infrastructure est planifiée, financée et mise en place au Canada »110. Spécifiquement en matière de transport en commun au Canada, le gouvernement fédéral attire notre attention sur le fait que la nouvelle BIC « jouera un rôle clé pour ce qui est de définir et de bâtir l'infrastructure de transport en commun au Canada $»^{111}$. Par conséquent, nous devons noter que la BIC embauchera les experts nécessaires pour établir, conclure et exécuter des " ententes complexes relatives à l'infrastructure $»^{112}$. En fait, la BIC jouera un rôle majeur dans tout ce qui a trait à la conception, la construction et la gestion des infrastructures au Canada ${ }^{113}$.

Deuxièmement, les investissements privés et institutionnels joueront probablement un rôle majeur dans le fonctionnement et la réalisation des projets de la BIC. Étant donné l'ampleur de leurs investissements et pour des raisons d'efficacité et de coordination, ces investisseurs vont-ils demander que la planification stratégique de l'infrastructure au Canada soit faite principalement par le gouvernement fédéral et que soit exclue l'application des lois provinciales?

Troisièmement, la BIC, pour faciliter effectivement l'exclusion de l'application des lois provinciales, ne sera-t-elle pas tentée (ou à la demande du secteur 
privé) d'utiliser son statut de mandataire de Sa Majesté du Chef du Canada pour exécuter elle-même des projets d'infrastructure? ${ }^{114}$ Cette question des infrastructures publiques préoccupe le Québec, notamment sous l'angle de la lutte contre les changements climatiques et de la nécessité de s'adapter à ceux-cí115.

De façon pratique, en matière de construction et d'entretien des infrastructures, le Québec intervient déjà au moins sur trois plans. D'abord, il a adopté en 2013 la Loi sur les infrastructures publiques ${ }^{116}$ dont la mission est d'établir « des règles de gouvernance en matière de planification des investissements publics en infrastructures de même qu'en matière de gestion des infrastructures publiques $»^{117}$. En vertu de cette loi, le Conseil du trésor propose annuellement au gouvernement « un plan des investissements publics des organismes du gouvernement en matière d'infrastructures portant sur 10 ans $»^{118}$. Quant à la Société québécoise des infrastructures, créée par cette même loi, elle vise à « soutenir les organismes publics dans la gestion de leurs projets d'infrastructures publiques et de développer, maintenir et gérer un parc immobilier qui répond à leurs besoins $»^{119}$.

Ensuite, le 12 juin 2015, l'Assemblée nationale adoptait la Loi visant à permettre la réalisation d'infrastructures par la Caisse de dépôt et placement du Québec120. À cet effet, la CDPQ a créé, en vertu de la Loi (québécoise) sur les societés par actions ${ }^{121}$, une filiale appelée CDPQ Infra Inc. dont l'une des activités concerne les «planifications, opérations et financements d'infrastructures $»^{122}$. Cette filiale a été le promoteur du Projet de résean électrique métropolitain de transport collectif présenté au BAPE au cours de 2016123.

Enfin, le 10 décembre 2016, l'Assemblée nationale du Québec adoptait la Loi sur Transition énergétique Québec ${ }^{124}$ créant l'organisme appelé Transition énergétique Québec (TEQ) dont la mission est de « [...] soutenir, de stimuler et de promouvoir la transition, l'innovation et l'efficacité énergétique et d'en assurer une gouvernance intégrée. Elle coordonne la mise en œuvre de l'ensemble des programmes et des mesures nécessaires à l'atteinte des cibles en matière énergétique déterminées par le gouvernement et en assure le suivi. Dans le cadre de sa mission, elle élabore le plan directeur en transition, innovation et efficacité énergétiques, dans une perspective de développement économique responsable et durable $»^{125}$. Dans l'élaboration de son Plan directeur en transition, innovation et efficacité énergétiques, le TEQ abordera d'une façon ou d'une autre la question des infrastructures ${ }^{126}$.

Compte tenu, entre autres choses, des immenses sommes d'argent dont elle disposera et des ententes complexes auxquelles participeront plusieurs parties, la question de la BIC est préoccupante si on regarde les enjeux qu'elle soulève : la place du secteur privé, les possibilités d'empiétement dans des champs de compétence des États fédérés, les stratégies à utiliser pour lutter contre les changements climatiques et l'adaptation à ceux-ci et, surtout, les processus décisionnels en cause, entre autres, ceux ayant trait à l'évaluation environnementale.

La BIC soulève aussi la question délicate du «pouvoir de dépenser » du gouvernement fédéral ${ }^{127}$ et celle ayant trait à la capacité juridique des municipalités pour établir des relations avec celui-ci ${ }^{128}$.

\section{Conclusion}

En mars 2017, en insérant une disposition préliminaire dans la Loi sur la qualité de l'environnement, l'Assemblée nationale marquait clairement son intention d'établir un lien entre la Loi sur le développement durable et la Loi sur la qualité de l'environnement (LDD - LQE), plus particulièrement avec le régime d'autorisation environnementale prévu par la LQE. Ce faisant, l'Assemblée nationale favorise grandement l'effectivité des principes de développement durable et de tout le droit qui entoure la mise en application des nouveaux concepts juridiques et valeurs sociétales véhiculés par ces principes. Les trois « terrains d'application » nous aident cependant à décrypter la complexité relative à la nature, la portée et l'applicabilité de cette nouvelle disposition législative.

Du côté de l'applicabilité de ce lien LDD - LQE dans le contexte de la fédération canadienne, ce sont les principes du « fédéralisme coopératif » qui doivent prévaloir. En effet, les projets qui sont assujettis au régime d'autorisation environnementale ont souvent des répercussions majeures sur le territoire québécois. D'où l'importance d'utiliser l'expertise québécoise en matière d'évaluation environnementale.

Les décrets adoptés par le gouvernement québécois au terme du régime d'autorisation environnementale comptent parmi les instruments juridiques qu'il utilise pour rendre ses décisions. Or, le contenu des 
décrets devra être reconfiguré pour traduire les nouvelles réalités juridiques et sociétales. Les contours des nouveaux concepts juridiques seront difficiles à établir, d'où l'importance de faire participer l'ensemble des parties prenantes. En fait, comme le souligne le professeur Daniel Mockle, on devra tenir compte « du caractère polycentrique de la gouvernance où de nombreux acteurs plus ou moins associés sont appelés à élaborer de nouveaux dispositifs publics (codes volontaires, chartes, plans, ententes de gestion) »129. Et l'un des volets du rôle de l'État québécois est de favoriser la mise en place de cette " gouvernance polycentrique", laquelle sera de nature à contribuer à l'effectivité du droit relié au lien LDD - LQE.

\section{NOTES}

1 Cette modification de la Loi sur la qualité de l'environnement a été faite par l'article 1 du projet de loi 102 (Loi modifiant la Loi sur la qualité de l'environnement afin de moderniser le régime d'autorisation environnementale et modifiant d'autres dispositions législatives notamment pour réformer la gouvernance du fonds vert), sanctionné le 23 mars 2017.

2 Loi sur le développement durable, RLRQ c. D-8.1.1. À noter que, de son côté, le Parlement fédéral a adopté la Loi fédérale sur le développement durable, LC 2008, c. 33.

3 Loi sur la qualité de l'environnement, RLRQ c Q -2.

4 Supra, note 1.

5 Loi d'interprétation, RLRQ c 1-16, articles 40, 41 et 41.1.

6 Voir Stratégie gouvernementale de développement durable (2015-2020), document rendu public par le gouvernement québécois en août 2015, p. 30. De son côté, le gouvernement fédéral a adopté la Stratégie fédérale de développement durable 2016-2019 (mis à jour, printemps 2017).

7 Voir R c. Hydro-Québec [1997] 3 R.C.S. 213.

8 Commentaires de la professeure Paule Halley dans « L'environnement au Québec » (1997), 15 Bulletin CCH; voir aussi Friends of the Oldman River Society c. Canada (Ministre des Transports) (1992) 1 R.C.S. 3.

9 Voir les articles 91 et 92 de la Loi constitutionnelle de 1867. À noter que sur le plan fédéral, la Loi canadienne sur la protection de l'environnement (1999), LC 1999, c 33 est une loi « pivot» dans le corpus du droit environnemental fédéral au même titre que la Loi sur la qualité de l'environnement sur le plan québécois.

10 Loi sur le développement durable, supra, note 2, art. 1. Voir Denis Bourque (2013). L'impact de la Loi sur le développement durable sur le concept de développement. Dans P.A Tremblay et S. Tremblay (dir.), Penser le développement, Chicoutimi, UQAC/GRIR, p. 51.

11 Loi sur le développement durable, supra, note 2, art. 1.

12 Ibid., art. 2.

13 Ibid., art. 3

14 Ibid., art. 4.

15 Wallot c. Québec (Ville de), 2011 QCCA 1165. [Requête pour autorisation de pourvoi à la Cour suprême rejetée. (C.S. Can., 2012-02-02, 34440)].

16 Ibid., paragr. 27. Voir dans le même sens Courses automobiles Mont-Tremblant Inc. c. Iredale 2013 QCCA 1348, paragr. 143.

17 Voir supra, note 6, Stratégie gouvernementale de développement durable (2015-2020).

18 Voir la Loi sur le développement durable, supra, note 2, art. 6. À noter que la majorité des principes de développement durable inscrits à l'article 6 sont des " principes de gestion ». Paule Halley et Denis Lemieux (2009). La mise en œuvre de la Loi québécoise sur le développement durable : un premier bilan. Dans Conférence des juristes de l'État 2009. Montréal, Québec : Éditions Yvon Blais, p. 103-114. Voici les 16 principes de développement durable qui apparaissent à l'article $6:$ : santé et qualité de vie, équité et solidarité sociales, protection de l'environnement, efficacité économique, participation et engagement, accès au savoir, subsidiarité, partenariat et coopération intergouvernementale, prévention, précaution, protection du patrimoine culturel, préservation de la biodiversité, respect de la capacité de support des écosystèmes, production et consommation responsables, pollueur payeur, internalisation des coûts ».

19 Voir Loi sur le développement durable, supra, note 2, art. 15 à 17.

20 Voir Loi sur le développement durable, supra, note 2, art. 34. À noter que l'adoption « d'indicateurs de développement durable » pour « surveiller et mesurer les progrès réalisés au Québec en matière de développement durable » peut aider le commissaire au développement durable à assumer ses responsabilités. Supra, note 2, art. 12. 
21 Supra, note 2, art. 19. Dans la Stratégie gouvernementale de développement durable 2015-2020, le gouvernement souligne que sa stratégie s'inscrit dans le plein respect des cadres légaux et législatifs du Québec. À cet effet, il rappelle l'existence de l'article 46.1 de la Charte des droits et libertés de la personne, supra, note 6, p. 14. Nous devons faire la distinction suivante: l'article 46.1 de la Charte attribue un droit (relatif) à toute personne, tandis que la majorité des principes de développement durable inscrits à l'article 6 de la Loi sur le développement durable sont plutôt, comme nous l'avons vu (supra, note 18), des « principes de gestion». À noter que le mot «loi » indiqué à l'article 46.1 inclut un règlement, un décret, une ordonnance ou un arrêté en conseil [art. 56 (3) Charte des droits et libertés de la personne].

22 Voir Paule Halley et Denis Lemieux (2009). La mise en œuvre de la Loi québécoise sur le développement durable : un premier bilan. Dans Conférence des juristes de l'État 2009, supra, note 18, p.126.

23 Ibid., p. 127.

24 Ibid.

25 Ibid.

26 Ibid. p. 128.

27 Saint-Luc-de-Vincennes (Municipalité de) c. Compostage Mauricie Inc., J.E. 2008-420, par. 46-47 (rapporté par Paule Halley et Denis Lemieux, supra, note 18, p. 129). La Cour suprême du Canada a refusé le 31 juillet 2008 la permission d'appel de ce jugement.

28 Baker c. Canada (Ministre de la Citoyenneté et de l'Immigration), [1999] 2 R.C.S. 817.

29 Pour des exemples, voir 114957 Can. Ltée (Spraytech arrosage) c. Hudson [2001] 2 R.C.S. 241 et Ciment du Saint-Laurent Inc. c. Barrette, 2008 CSC 64.

30 Voir Loi sur le ministère du Développement durable, de l'Environnement et des Parcs, RLRQ c M-30.001, art. 10. Afin d'éviter toute confusion, notons que le ministre et le ministère du Développement durable, de l'Environnement et des Parcs sont désignés sous le nom de ministre et de ministère du Développement durable, de l'Environnement et de la Lutte contre les changements climatiques, Décret 374-2014 du 24 avril 2014 (2014) 146 G.O. 2, 1878. Notons également que ce même ministre est responsable de la gestion du Fonds vert créé par l'article 26 de la Loi sur le développement durable.

31 Loi sur la qualité de l'environnement, supra, note 3, art. 20 et suivants.

32 Ibid., art. 31.10 et suivants.

33 Ibid., 31.42 et $\mathrm{s}$.

34 Ibid., art. 31.74 et s.

35 Ibid., art. 46.1 et s.

36 Ibid., art. 53.1 et s.

37 Voir l'article 31.1 de la LQE, supra, note 3 et le Règlement sur l'évaluation et l'examen des impacts sur l'environnement, RLRQ c Q-2, r 23.

38 Supra, note 2.

39 Voir Bureau d'audiences publiques sur l'environnement (BAPE) (13 mai 1985). Programme de stabilisation des berges du lac Saint-Jean, rapport d'enquête et d'audience publique (rapport 19). Repéré à www.bape.gouv.qc.ca/sections/ rapports/publications.

40 Ibid., p. 2.7.

41 Ibid.

42 Ibid., p. 1.9, 1.10, 1.20, 2.6.

43 Ibid., p. 1.19

44 Ibid., p. 2.7, 2.8, 8.15.

45 Voir Rio Tinto Alcan (2016). Programme de stabilisation des berges du lac Saint-Jean 2017-2026 - Étude d'impact sur l'environnement, déposé en août 2016, Bureau d'audiences publiques sur l'environnement.

46 Ibid., p. 2-5 à 2-7.

47 Ibid., p. 2-5.

48 Ibid., p. 2-6.

49 Ibid., p. 2-7. 
50 Entente de principe - modèle de gestion participative du lac Saint-Jean, Entente de principe entre la municipalité régionale de comté de Lac-Saint-Jean-Est et la municipalité régionale de comté de Maria-Chapdelaine et la municipalité régionale de comté du Domaine-du-Roy et Pekuakamiulnuatsh Takubikan et Rio Tinto Alcan (ci-après appelée «Entente de principe d'avril 2017 », déposée au Bureau d'audiences publiques sur l'environnement).

51 Voir Bureau d'audiences publiques sur l'environnement (septembre 2017). Programme de stabilisation des berges du lac SaintJean 2017-2026 - Rapport d'enquête et d'audience publique (rapport 337) (ci-après appelé BAPE 2017), p. 3, 13 à 15, 27 à 29; Voir aussi Tremblay, L. (2017, 20 septembre), Le BAPE retient l'entente des parties prenantes. Le Quotidien. Repéré à www.lequotidien.com/actualités.

52 Voir notamment Riverains, Lac Saint-Jean 2000, D'une rive à l'autre du lac! D'un bord à l'autre des berges!, mémoire déposé au BAPE en juin 2017 et Un lac pour tous, mémoire déposé au BAPE par le Comité des parties prenantes de la gestion durable du lac Saint-Jean, 13 juin 2017.

53 Supra, BAPE 2017, note 51, p. 13 et s.

54 Supra, note 51 , p. 35 et s.

55 Supra, note 51, p. 29.

56 Supra, note 51, p. 13.

57 Décret délivré en vertu de l'article 31.1 de la LQE, supra, note 37.

58 Supra, note 15, paragr. 24.

59 Voir les contributions au titre de «État fiduciaire et gardien du patrimoine », dans Paule Halley (dir.) avec la coordination de Julia Sotousek, L'environnement, notre patrimoine commun et son État gardien. Aspects juridiques, nationaux, transnationaux et internationaux, Montréal, Québec : Éditions Yvon Blais, 2012, p. 159-479; Gouvernement du Québec, Orientations du ministère de l'Énergie et des Ressources naturelles en matière d'acceptabilité sociale - Livre vert, février 2016, p. 25; Bureau d'audiences publiques sur l'environnement, Développement durable de l'industrie des gaz de schiste an Québec - Rapport d'enquête et d'audience publique (rapport 273), février 2011, p. 245. Dans le Rapport du Vérificateur général du Québec à l'Assemblée nationale pour l'année 2008-2009, t. II, avril 2009, p. 2-7, le Vérificateur général du Québec s'exprime ainsi : «L'activité minière est avant tout influencée par l'entreprise privée et le marché financier. L'État est cependant tenu de jouer un rôle de fiduciaire du bien commun que sont nos ressources naturelles. Les enjeux auxquels il doit faire face sont autant d'ordre économique que social et environnemental. »

60 Voir article 1 de la Loi affirmant le caractère collectif des ressources en eau et visant à renforcer leur protection, RLRQ c C-6.2; voir également L'environnement, notre patrimoine commun et son État gardien, supra, note 59.

61 Voir le 4e « considérant» de la Loi affirmant le caractère collectif des ressources en eau et visant à renforcer leur protection, supra, note 60 et L'environnement, notre patrimoine commun et son État gardien, supra, note 59.

62 Orientations du ministère de l'Énergie et des Ressources naturelles en matière d'acceptabilité sociale, supra, note 59; voir Bureau d'audiences publiques sur l'environnement (décembre 2013). Projet d'ouverture et d'exploitation d'une mine d'apatite à Sept-Îles Rapport d'enquête et d'audience publique (rapport 301), p. 119; voir Développement durable de l'industrie des gaz de schiste au Québec - Rapport d'enquête et d'audience publique, supra, note 59, p. 245; Rapport BAPE 2017, supra, note 51, p. 17-18; voir P. Batelier et M.E Maillé, Acceptabilité sociale : sans oui, c'est non, Montréal, Écosociété, 2017. Voir Entente de principe d'avril 2017, supra, note 50, art. 1.1 et 1.2.3.

63 Voir l'arrêt Ciment du Saint-Laurent Inc. c. Barrette, supra, note 29, p. 6 et paragr. 80. Voir également l'article 4 de la Loi affirmant le caractère collectif des ressources en eau et visant à renforcer leur protection, supra, note 60 .

64 Voir Entente de principe d'avril 2017, supra, note 50, art. 1.2.3. Voir le «1 $1^{\text {er }}$ considérant » de la Loi sur les mines, RLRQ c M-13.1 et le " $1^{\text {er }}$ considérant» de la Loi sur l'aménagement durable du territoire forestier, RLRQ c A-18.1. Voir aussi l'affirmation du Comité des parties prenantes de la gestion durable du lac Saint-Jean, dans le rapport BAPE 2017, supra, note 51, p. 15. Ces nouveaux concepts juridiques et valeurs environnementales se raccrochent directement ou indirectement aux 16 principes de développement durable énoncés dans la LDD, supra, note 18. Dans son rapport de juin 2017, Un environnement sain, des Canadiens et une économie en santé : renforcer la Loi canadienne sur la protection de l'environnement (1999), le Comité permanent de l'environnement et du développement durable de la Chambre des communes recommande que le « préambule de la LCPE soit modifié de manière à reconnaître le droit à un environnement sain ", p. 8. Également, du côté fédéral, voir le projet de loi C-57 (Loi modifiant la Loi fédérale sur le développement durable (déposé le 19 juin 2017) qui change la Loi fédérale sur le développement durable en intégrant, entre autres choses, dans celle-ci des principes de développement durable qui recoupent, en partie, ceux prévus à l'article 6 de la Loi (québécoise) sur le développement durable.

65 Supra, note 15, paragr. 25 et 26.

66 Supra, BAPE 2017, note 51, p. 28.

67 Rio Tinto Alcan, Étude d'impact sur l'environnement, supra, note 45, p. 2-7. 
68 Ibid.

69 Ibid. Voir également BAPE 2017, supra, note 51, p. 28.

70 Rio Tinto Alcan, Étude d'impact sur l'environnement, supra, note 45, p. 2-7.

71 Voir Chaire en éco-conseil (mai 2015). Rapport d'analyse de développement durable du Programme de stabilisation des berges du lac Saint-Jean de Rio Tinto Alcan division Énergie Électrique. Université du Québec à Chicoutimi.

72 Ibid. p. 62-64. Voir Tremblay, L. (2017, 22 avril). Gestion du lac Saint-Jean : Rio Tinto garde la décision finale. Le Quotidien. Repéré à https://www.lequotidien.com/actualités. Le professeur Claude Villeneuve réaffirme la position de la Chaire en éco-conseil. À noter que la grille d'analyse de développement durable de la Chaire en éco-conseil a reçu une reconnaissance internationale; voir Villeneuve, C. (2017, 28 juillet). Reconnaissance internationale. Le Quotidien. Repéré à https://www.lequotidien/chroniques/Claude-Villeneuve. La position de la Chaire en éco-conseil est rapportée dans le rapport BAPE 2017, supra, note 51, p. 27.

73 Supra, les notes 21 à 27 et le texte correspondant.

74 Rapporté par le BAPE 2017, supra, note 51, p.15.

75 Rio Tinto Alcan, Étude d'impact sur l'environnement, supra, note 45, p. 2-7. Ce point est rapporté par le BAPE 2017, supra, note 51, p. 28.

76 Voir BAPE 2017, supra, note 51, p. 39.

77 Ibid., p.41. La création des organismes de bassins versants découle des pouvoirs conférés au ministre du Développement durable, de l'Environnement et des Parcs par les articles 13 et 14 de la Loi affirmant le caractère collectif des ressources en eau et visant à renforcer leur protection, supra, note 60 .

78 BAPE 2017, supra, note 51, p. 14. Voir David Massell (2011). Quebec Hydropolitics. McGill Queen’s University Press.

79 Dans son mémoire présenté au BAPE en juin 2017, Riverains, Lac Saint-Jean 2000 a fait valoir l'argument basé sur l'article 46.1 de la Charte des droits et libertés de la personne, supra, note 52, p. 24 et annexe 6; voir également S. Thériault et D. Robitaille (2011), Les droits environnementaux dans la Charte des droits et libertés de la personne du Québec : pistes de réflexion, Revue de droit de McGill, 57(2), p. 211.

80 Dans son rapport le BAPE précise que les «personnes qui s'estimeraient lésées par la gestion du niveau du lac Saint-Jean que pratique le promoteur ont la possibilité, faute d'en arriver à une solution négociée avec ce dernier, d'entreprendre des recours judiciaires, et ce, à titre individuel ou collectif ». Supra, note 51, p. 31. Dans cette optique, si une personne ou un groupe décidait d'exercer une poursuite judiciaire, il serait probablement opportun d'étudier la possibilité d'utiliser le recours mentionné dans l'arrêt Ciment du Saint-Laurent, supra, note 29. Il importe également de noter que dans l'arrêt Ciment du Saint-Laurent, l'argument de la compagnie, selon lequel la Loi spéciale CSL adoptée par la législature du Québec en 1952 pour régir ses activités lui conférant l'immunité à l'égard des poursuites en dommage-intérêts en relation avec ses activités industrielles, n'a pas été retenu. Supra, note 29, paragr. 97 et 98.

81 Voir BAPE 2017, supra, note 51, p. 15. Dès 1985, le BAPE considérait « qu’il faudrait soutenir le caractère collectif de la gestion des usages du lac et assurer un support institutionnel à l'interaction et à la coordination des principaux partenaires $[\ldots] »$, supra, note 39 , p. 8-12.

82 Voir l'article 1 de la Loi sur le développement durable, supra, note 2; voir aussi l'article 13 de la Loi affirmant le caractère collectif des ressources en eau et visant à renforcer leur protection, supra, note 60 .

83 Marie-Claude Prémont (2016). Le cadre juridique des droits de production hydroélectrique au Saguenay-Lac-Saint-Jean. Organisations et territoires, 25(3), p. 77-79; David Massell (2011). Quebec Hydropolitics, supra, note 78.

84 Le premier projet concerne, d'une part, le dédoublement du réseau actuel de Trans Mountain Pipeline, qui s'étend sur 1147 kilomètres entre Edmonton, Alberta, et Burnaby, Colombie-Britannique, et, d'autre part, l'agrandissement de son terminal maritime Westridge (TMW) de la Baie Burrard. C.P. 2016-1069, 29 novembre 2016 (Trans Mountain). Le deuxième projet vise le remplacement d'un oléoduc sur une distance d'environ $1096 \mathrm{~km}$ par un nouveau pipeline qui relie Hardisty, en Alberta, à Gretna, au Manitoba, et se rend jusqu’à Superior, dans le Wisconsin, aux États-Unis. C.P. 20161048, 25 novembre 2016. (Remplacement de la canalisation no 3). Voir Shields, A. (2016, 30 novembre). Ottawa approuve deux importants projets d'oléoducs. Le Devoir. Repéré à www.ledevoir.com/actualités. Quant au projet Énergie Est, il a été abandonné par le promoteur, la compagnie TransCanada. Rappelons que le projet Énergie Est visait essentiellement la construction d'un pipeline d'une longueur de 4800 kilomètres entre l'Alberta et le port de Saint John au Nouveau-Brunswick. Ce pipeline traversait donc plusieurs provinces; il utilisait le territoire québécois sur une longueur de près de 800 kilomètres, en passant sur les terres agricoles et en traversant tout près de 650 cours d'eau. Voir Shields, A. (2017, 6 octobre). Énergie Est : la logique d'affaires l'emporte. Le Devoir. Certains experts prétendent que le projet Énergie Est pourrait bien revenir. Voir La Presse canadienne. (2017, 7 octobre). L'abandon d'Énergie Est laisse peu de marge aux autres projets, selon un analyste. Le Soleil. Repéré à https://www.lesoleil.com

85 Voir notamment les articles 52 et suivants de la Loi sur l'Office national de l'Énergie, LRC 1985, c N -7. 
86 Coastal First Nations v. British Columbia (Environment), 2016 BCSC 34.

87 Ibid., paragr. 25 à 27.

88 Ibid., paragr. 57 à 77. Voir aussi O’Callaghan K. et Romano, Z. (2016, 26 janvier). Évaluation environnementale du projet de pipeline Northern Gateway : la Colombie-Britannique conserve l'obligation de consulter et le pouvoir d'imposer des conditions supplémentaires. Bulletin Affaires autochtones, p. 1-3. Repéré à www.fasken.com/fr/publications.

89 Éditorial (2017, 4 juin). Globe editorial : On pipelines, Ottawa must have the final say. The Globe and Mail. Repéré à https://www.theglobeandmail.com.

90 Loi constitutionnelle de 1867, art. 91, paragr. introductif.

91 Ibid.

92 Ibid., paragr. 92(10) (c). Voir H. Brun, G. Tremblay et E. Brouillet (2014). Droit constitutionnel, 6e éd., Montréal, Québec : Éditions Yvon Blais, p. 428 et 588. Pour l'application du pouvoir déclaratoire fédéral au domaine de l'énergie nucléaire, voir D. Bourque (1990). L'énergie nucléaire et le droit: les autorisations, l'environnement, les contrôles judiciaires et politiques : étude comparative, Montréal, Québec : Éditions Yvon Blais, p. 625 et suivantes.

93 Voir Droit constitutionnel, supra, note 92.

94 Cornellier, M. (2017, 4 juillet). La poutre pétrolière. Le Devoir. Repéré à www.ledevoir/politique/canada. Shields, A. (2017, 28 juin). Ottawa ouvre une future zone de protection marine aux pétrolières. Le Devoir. Repéré à www.ledevoir.com/environnement/actualités-sur-l-environnement.

95 Comité des experts pour l'évaluation des impacts au Canada. (2017). Bâtir un terrain d'entente : une nouvelle vision pour l'évaluation des impacts au Canada - le rapport final du comité d'experts pour l'évaluation des processus d'évaluation environnementale.

96 Comité d'experts sur la modernisation de l'Office national de l'énergie. (2017). Rapport. Progresser ensemble - Favoriser l'avenir énergétique propre et sécuritaire du Canada et voir les Rapports de la commissaire à l'environnement et au développement durable du Parlement du Canada, automne (2017). Voir La Presse canadienne. (2017, 4 octobre). Le Canada n'est pas prêt, dit la commissaire à l'environnement : le pays a raté et ratera sans doute encore ses cibles de réduction de gaz à effet de serre déplore aussi Julie Gelfand. Le Devoir. Repéré à https://www.ledevoir.com/environnement/ actualites-sur-l-environnement.

97 Daniel Robitaille (2015), Le transport interprovincial sur le territoire local : vers un nécessaire équilibre, Revue d'études constitutionnelles, 20(1), p. 75-115.

98 Voir Infrastructure Canada. (2017). Plan investir dans le Canada. Repéré à www.infrastructure.gc.ca.

99 Dans le cadre du Plan investir dans le Canada, le ministre fédéral de l'Infrastructure et des Collectivités, monsieur Amarjeet Sohi, a écrit une lettre aux provinces et aux territoires à propos des ententes bilatérales intégrées. On peut lire celle adressée le 6 juillet 2017 au ministre québécois des Finances, monsieur Carlos Leitão. Plan Investir dans le Canada : lettres à propos des ententes bilatérales intégrées - Québec. Repéré à www.infrastructure.gc.ca.

100 La Loi sur la Banque de l'infrastructure du Canada fait partie d'une loi plus considérable intitulée Loi portant exécution de certaines dispositions du budget déposé au Parlement le 22 mars 2017 et mettant en cuvre d'autres mesures, sanctionnée le 22 juin 2017, L.C, 2017, ch. 20.

101 Voir Loi sur la Banque de l'infrastructure du Canada, supra, note 100, art. 5(1).

102 Ibid., art. 6.

103 Ibid., art. 7(1)h).

104 Ibid., art. 18h). Notons que la Banque peut elle-même investir dans les projets d'infrastructures ou recommander que des prêts soient consentis à l'égard de tels projets [Ibid., art. 7(1)b) et art. 22(1)]. Notons que le ministre fédéral des Finances peut verser à la Banque des sommes ne dépassant pas globalement 35 milliards, (ibid., art. 23). Ceci ne comprend pas évidemment l'apport des investissements d'investisseurs du secteur privé et d'investisseurs institutionnels.

105 Ibid., art. 5(4)c).

106 Ibid., art. 5(4)d).

107 Les propos du professeur Taillon sont rapportés dans Foisy, P.V. (2017, 31 mai). Banque de l'infrastructure : Québec « exige » des modifications. Radio-Canada. Repéré à www.ici.radio-canada.ca/nouvelles. Voir Loi (fédérale) d'interprétation, LRC 1985, c I -21, article 17.

108 Supra, note 107. Voir également P. Garant (2010). Droit administratif (6e éd.), Montréal, Québec : Éditions Yvon Blais, p. $145-150$.

109 Gouvernement du Canada, 2016 Un plan pour faire progresser la classe moyenne - Énoncé économique de l'automne 2016, p. 32. 
110 Gouvernement du Canada, Le Budget de 2017 - Bâtir une classe moyenne forte, p. 133.

111 Ibid., p. 135.

112 Supra, note 109. Voir aussi Gouvernement du Canada (2016). Assurer la croissance de la classe moyenne, (budget de 2016), p. 101.

113 Supra, note 109.

114 Voir Loi sur la Banque de l'infrastructure du Canada, supra, note 99, art. 5(4)d) et 18h). Gouvernement du Canada, Réaliser un avenir durable - Stratégie fédérale de développement durable 2016-2019 (mise à jour, printemps 2017), supra, note 6, p. 8, réfère spécifiquement à la BIC.

115 Gouvernement du Québec (2012). Le Québec en action vert 2020 - Plan d'action 2013-2020 sur les changements climatiques, p. 38-39; Le Québec en action vert 2020 - Stratégie gouvernementale d'adaptation aux changements climatiques 2013-2020, p. 9.

116 Loi sur les infrastructures publiques, RLRQ c I-8.3.

117 Ibid., art. 1.

118 Ibid., art. 6.

119 Ibid., art. 25. Voir aussi Conseil du trésor (mars 2017). Les infrastructures publiques du Québec - Plan québécois des infrastructures 2017-2027 - Plans annuels de gestion des investissements publics en infrastructures 2017-2018. Repéré à https://www.tresor.gouv.qc.ca/file.adm/PDF/budget_depenses/17-18/InfrastructuresPubliques.pdf.

120 Projet de loi no 38 devenu chapitre 17 des lois de 2015.

121 Loi sur les sociétés par actions, RLRQ c S-31.1.

122 Registraire des entreprises. Repéré à www.enregistreentreprises.gouv.qc.ca.

123 Bureau d'audiences publiques sur l'environnement (2016). Projet de réseau électrique métropolitain de transport collectif - Rapport d'enquête et d'audience publique (rapport no 331).

124 Loi sur transition énergétique Québec, RLRQ c T-11.02.

125 Ibid., art. 4, 7 à 17.

126 Voir Pierre Arcand. (26 juin 2017). Le ministre Arcand dévoile le premier Plan d'action de la Politique énergétique 2030 (communiqué de presse - Énergie). Ministère de l’Énergie et des Ressources naturelles. Repéré à www.mern.qc.ca.

127 Supra, Droit constitutionnel, note 92, p. 439.

128 Voir Loi sur le ministère du Conseil exécutif, RLRQ c M -30, art. 3.6.2 et 3.8.

129 Daniel Mockle (2008), La gouvernance publique et le droit. Dans Pierre Noreau (dir.), Le droit à tout faire : exploration des fonctions contemporaines du droit - (All Purpose Law : Exploring Contemporay Functions of Law). Québec, Québec : Les Éditions Thémis, p. 73. 